\title{
Porta Valbona a Urbino: la sua rappresentazione tra storia e restauro
}

Porta Valbona in Urbino: its representation between history and restoration

\author{
Laura Baratin $^{\text {a }}$, Alessandra Cattaneo ${ }^{\text {b }}$, Elvio Moretti $^{\mathrm{c}}$ \\ Scuola di Conservazione e Restauro - Dipartimento di Scienze Pure e Applicate - Università degli Studi di Urbino \\ Carlo Bo, Urbino, Italy \\ a laura.baratin@uniurb.it; balessandra.cattaneo@uniurb.it; celvio.moretti@uniurb.it
}

\begin{abstract}
The Porta Valbona study is part of a complex project of conservation and valorisation of the defensive walls of Urbino that the research group, of the School of Conservation and Restoration of the University of Urbino Carlo Bo, has developed in recent years. Built in 1621 it is the most important gate of the city both because it is connected to Via Mazzini, one of the main streets of the historic centre, and for its spectacular architectural appearance created for the wedding of Prince Federico Ubaldo della Rovere with Princess Claudia de Medici. The two eagles, in limestone, placed at the sides of the door, date back to the mid-eighteenth century and are the work of the Rimini architect Giovan Francesco Buonamici. It is also the only Gate of Urbino which has a monumental facing facing outwards, or towards Piazza del Mercatale. Despite having undergone several restorations and consolidations over the centuries, it has not been modified in its original appearance. Porta Valbona, together with the city walls, represents a real urban palimpsest, an exceptional case of sedimentation and stratification which, despite the events, still allows us to reconstruct its historical events. The applied design method was based on the following analyzes: a) urban analysis: knowledge of the characteristics and urban potential of the door; b) historical analysis: knowledge of the historical evolution and of the specific qualities of the door; c) geometric analysis: metric and architectural survey; d) material analysis, study of materials and forms of deterioration; e) structural analysis: identification of the morphological and constructive organization from the structural point of view. All the large amount of information obtained from the analysis was managed thanks to the use of GIS systems. Thus it was possible to identify the shape and character of the monument and its testimonial, constructive and architectural values were recognized. On the basis of an internal analysis of the cultural asset and an external analysis of the context in which it is located, it was possible to define the strengths, weaknesses, opportunities and threats.
\end{abstract}

Keywords: Urban door, historical center, restoration, knowledge.

\section{Porta Valbona e le sue vicende storiche}

La narrazione sulle trasformazioni storiche di questa Porta è strettamente legata alle origini della città di Urbino dal suo antico tracciato romano fino alla sistemazione odierna. La struttura urbana della città mutò sensibilmente quando,

intorno al tracciato romano, cominciarono a sorgere le contrade sulle pendici del primo colle, sul vertice e sulle falde del secondo, verso San Bartolo, San Paolo e Santa Maria e verso il Monte, Santa Lucia e Valbona. Nelle rare stam- 
pe dell'epoca la città venne sempre ripresa nelle vedute da oriente poichè da quel lato si proiettava la sua immagine più complessa. La prima porta venne realizzata in concomitanza con la costruzione delle mura di età federiciana a -2,67 $\mathrm{m}$ dal piano stradale attuale le cui fondazioni vennero alla luce durante alcuni scavi (Fig. 1).

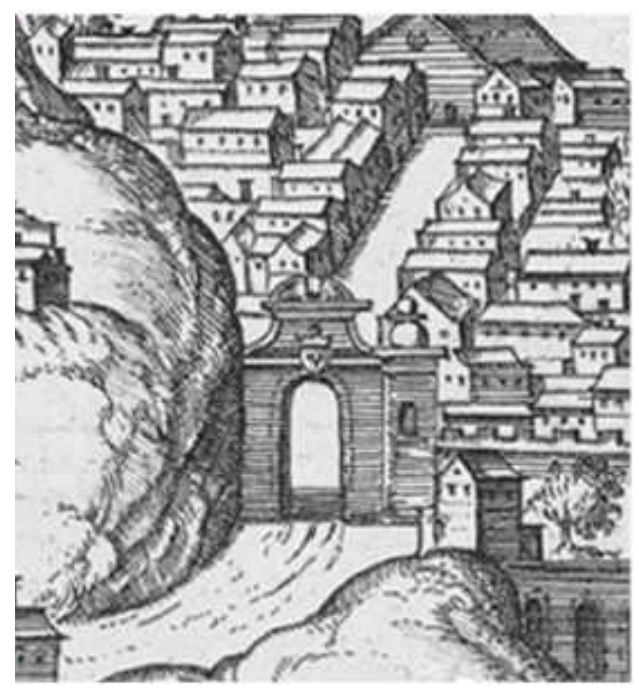

Fig. 1. Veduta di Urbino del 1587 di Braun \&Hogenberg (G. Cucco "Percorso iconografico" dal XV al XIX secolo”, Accademia Raffaello 1996).

La nuova porta fu eretta in occasione dei lavori di ristrutturazione delle mura e dei lavori di ampliamento del Palazzo Ducale. Dalle scarse fonti a disposizione sembra fosse composta da una semplice volta realizzata in muratura di laterizi e priva di qualsiasi ornamento. Nelle stampe del Cinquecento la città non fu più rappresentata da oriente ma da mezzogiorno, segno questo che la grande trasformazione rinascimentale era avvenuta. Si rilegge la verticale dei torricini del Laurana che cade sul piano del Mercatale e da qui parte la lunga direttrice obliqua, via di Valbona che risale fino al campanile della chiesa di San Francesco questa volta ripreso dalla sua faccia opposta. L'immagine della città venne quindi ribaltata sull'arco meridionale dove si concentrarono i simboli più significativi e i più importanti interessi.

Il paramento monumentale di Porta Valbona presenta una singolare architettura, volutamente scenografica: il suo "addobbo architettonico" fu realizzato nel 1621, in occasione delle nozze del principe Federico Ubaldo della Rovere con la principessa di Toscana Claudia de Medici. Per accogliere il corteo nuziale e gli sposi da Firenze, dove si erano uniti in matrimonio, in tutte le città dislocate lungo il percorso di rappresentanza furono allestiti archi trionfali arricchiti di figurazioni, per lo più opere precarie, fatta eccezione, a Urbino, per quanto riguarda questa Porta e del portico eretto sul fianco esterno del Duomo. Queste architetture furono realizzate nonostante si sapesse che sarebbero servite solo per il passaggio del corteo nuziale, e che gli sposi si sarebbero trattenuti solo per una notte. La porta venne adornata con un ordine gigante di colonne binate in stile tuscanico, due nicchie con statue, un fregio e un timpano spezzato. Nell'arco dei successivi tre secoli vennero eseguiti una serie di restauri che non modificarono radicalmente il monumento a eccezione dell'inserimento di due aquile in calcare massiccio nella prima metà del XVIII secolo in luogo delle edicole poste sopra le nicchie, le cui statue furono trafugate tra la fine del Seicento e l'inizio del Settecento (Fig. 2). Le due aquile araldiche poste ai lati del fastigio della porta furono realizzate a metà Settecento dall'architetto riminese Giovan Francesco Buonamici. Purtroppo sono andate perdute sia la statua allegorica della Fama, posta al centro del timpano spezzato, sia le due statue poste nelle nicchie ai lati della porta, raffiguranti una Umbro Suasso, mitico fondatore di Urbino, e l'altra Federico da Montefeltro.

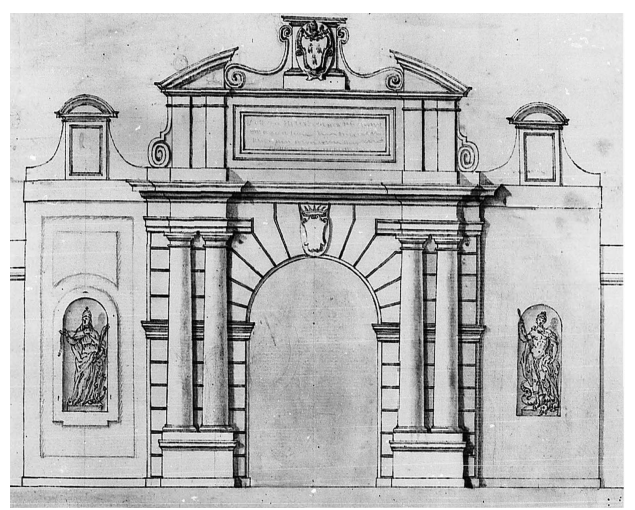

Fig. 2. Prospetto di Porta Valbona, Circolo di Carlo Fontana (1638-1710 ca.). 
L'area di confluenza intorno alla chiesa di San Francesco, che molti secoli dopo fu trasformata nella nuova piazza, cadeva proprio nel punto di massima oscillazione del tessuto urbano dove gli architetti del rinascimento avevano risolto la giunzione con la città preesistente saldando due eterogenee concatenazioni di spazi in un asse unificato che attraversava la città lungo la linea trasversale più breve da Valbona a Lavagine. Dall'ingresso tradizionale di Lavagine, integrato nella nuova struttura urbana, scorreva il flusso dei traffici e si diffondeva verso i punti di interesse commerciale delle zone circostanti. Il piazzale del Mercatale, la chiesa di San Francesco, la Cattedrale, la chiesa di San Girolamo, la chiesa di Santa Chiara, la Fortezza, le Porte, il Palazzo Ducale, le sequenze di spazi maggiori che connettevano questi epicentri funzionali e visivi, $i$ sistemi viari che incanalavano i principali flussi di traffico, costituivano la struttura organizzativa e formale della nuova città (Fig. 3).

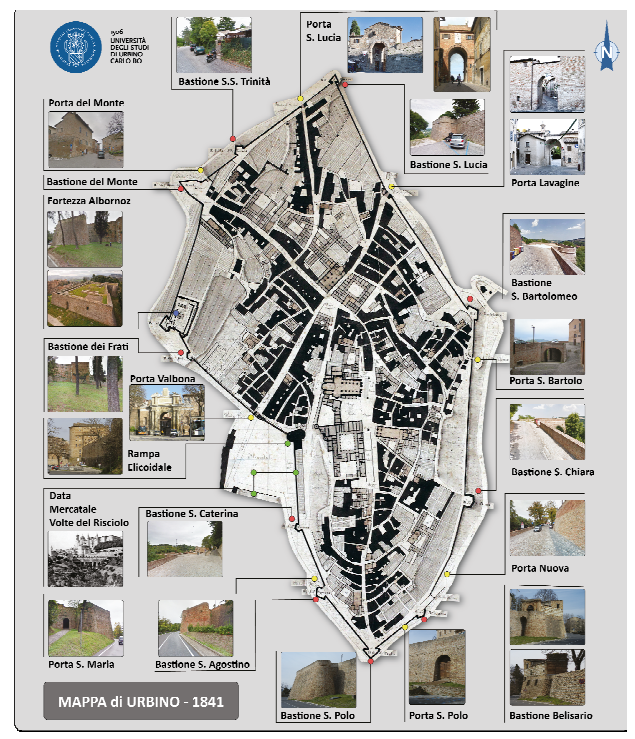

Fig. 3. Mappa di Urbino del 1841 con l'indicazione delle porte e del sistema fortificato (Archivio Scuola di Conservazione e Restauro).

Nel corso dei secoli vennero costruite delle abitazioni addossate al prospetto posteriore della Porta sfruttando gli ambienti dove le sentinelle, durante il periodo feltresco, montavano di guardia. Tra il gennaio del 1950 e il marzo del 1951 furono eseguiti alcuni restauri, durante i quali vennero ultimate delle piccole modifiche per la decorazione della Porta tra le quali l'inserimento di un'epigrafe sulla sommità del frontone che attestasse i restauri eseguiti in quegli anni. La redazione del testo fu affidata alla Curia Arcivescovile di Urbino e riportò la seguente frase ancor oggi visibile (Fig. 4):

\section{"ANNO JVBILAEI MCML AERE PVBBLICO PRINISTINI OPERIS AD EXEMPLAR RE- STITUTUM."}

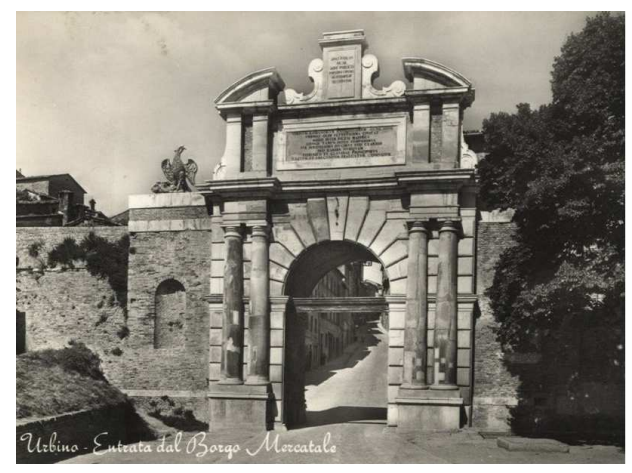

Fig. 4. Porta Valbona in una cartolina del 1959 (Archivio Scuola di Conservazione e Restauro).

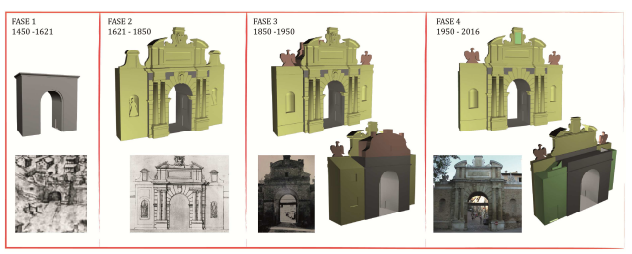

Fig. 5. Ricostruzione 3D delle diverse fasi costruttive che hanno interessato Porta Valbona.

Di quegli anni è anche il progetto che prevedeva l'abbattimento delle case addossate alla Porta per la realizzazione del camminamento esteso sulla parte orientale delle Mura fino alla Rampa Elicoidale di Francesco di Giorgio Martini.

Durante i restauri degli anni Cinquanta vennero eseguiti anche lavori di tipo strutturale, realizzando inoltre il camminamento, abbattendo le case in prossimità della Porta e collegando i due estremi di via Mazzini (Fig. 5). La funzione del piazzale Mercatale cambiò solo nella seconda metà del XX secolo con l'intervento dell'architetto Giancarlo De Carlo il quale rea- 
lizzò nella piazza sia un parcheggio sotterraneo articolato su due livelli che un ulteriore parcheggio di superficie con annessa la stazione degli autobus.

\section{Porta Valbona e gli interventi di restauro}

I primi restauri furono eseguiti da Mastro Marco Silvio Tommasini tra il 1755 e il 1756 e si concentrarono per lo più sulla parte ornamentale posizionando alcune pietre sotto le Aquile, realizzate dal Buonamici, per dar loro maggior stabilità e visibilità ed attuando degli interventi di consolidamento all'intera decorazione. Inoltre, sempre nell'ambito del medesimo restauro, vennero realizzate le armature della muraglia ai fianchi della Porta. Altri interventi furono fatti da Giuseppe Corsini nel 1825 per la sistemazione degli stemmi di Urbano VIII che coinvolsero anche Porta Lavagine e nel 1875 ulteriori interventi vennero svolti nella parte monumentale esterna della Porta. Dai documenti reperiti all' Archivio di Stato di Urbino risulta una perizia, datata 17 ottobre 1873, effettuata dell'Ufficio Tecnico del Comune di Urbino che evidenziava le gravi condizioni in cui Porta Valbona verteva e indicava che per tal motivo si provvedesse con estrema rapidità a un restauro di tipo strutturale in tutta la parte ornamentale all'infuori delle decorazioni, in pietra calcarea del Furlo, che risultavano comunque in discreto stato conservativo. Nonostante i lavori occorsi però, nel 1784, ci fu la necessità di una nuova perizia per dei lavori addizionali che furono eseguiti anch'essi con celerità e per i quali furono stanziate ben 252,65 lire. Sempre da fonti d'archivio il 27 settembre 1873 l'Ufficiale Tecnico del Comune di Urbino stilò un progetto di riduzione della pubblica Fonte di Valbona dove spiegò chiaramente le motivazioni dell'intervento sostenendone la portata innovatrice dal punto di vista estetico ma soprattutto da quello funzionale poiché la fonte era adibita per lo più ad abbeveratoio per gli animali date le sue ampie dimensioni (Fig. 6). Al progetto preliminare del 1873 seguì, nel 1875, il progetto esecutivo con indicazione dei lavori eseguiti e delle spese sostenute (Fig. 7).

Nella prima metà del Novecento vi furono una serie di interventi sulle pietre di base delle co- lonne sia a destra che a sinistra (1936-1937), dei paramenti in arenaria e delle murature (1950). Nei faldoni dell'Archivio del Comune di Urbino è stata raccolta tutta la documentazione sia progettuale che fotografica dei consolidamenti e trattamenti protettivi, delle micro cuciture delle parti in pietra e delle stuccature degli elementi lapidei e murari eseguiti nel 1985-1986 e successivamente nel 1995 dalla Ditta Carli su progetto di G. Croci (Fig. 8).

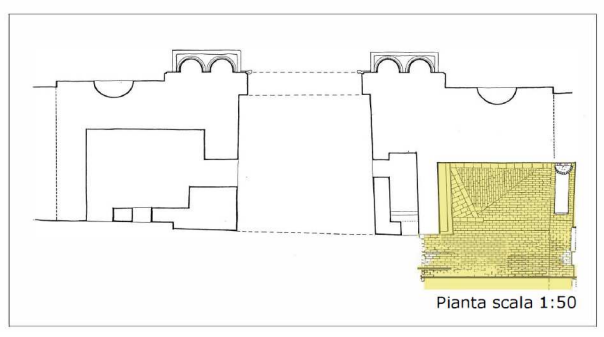

Fig. 6. Planimetria di Porta Valbona allo stato attuale con indicazione della Fonte (Archivio del Comune di Urbino).

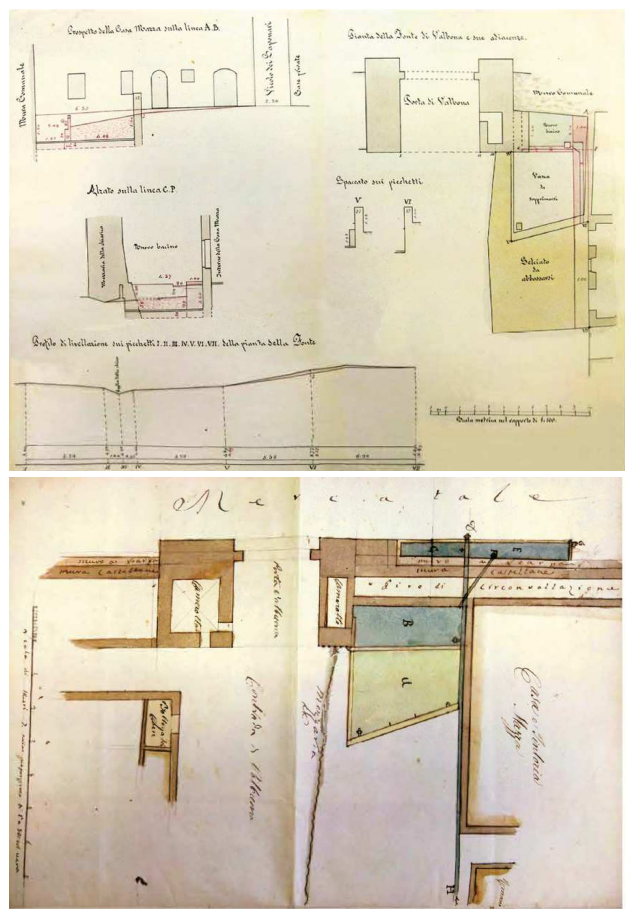

Fig. 7. Progetto di riduzione della pubblica Fonte di Porta Valbona nella sua fase preliminare sopra e in quella esecutiva sotto (Archivio di Stato di Urbino). 


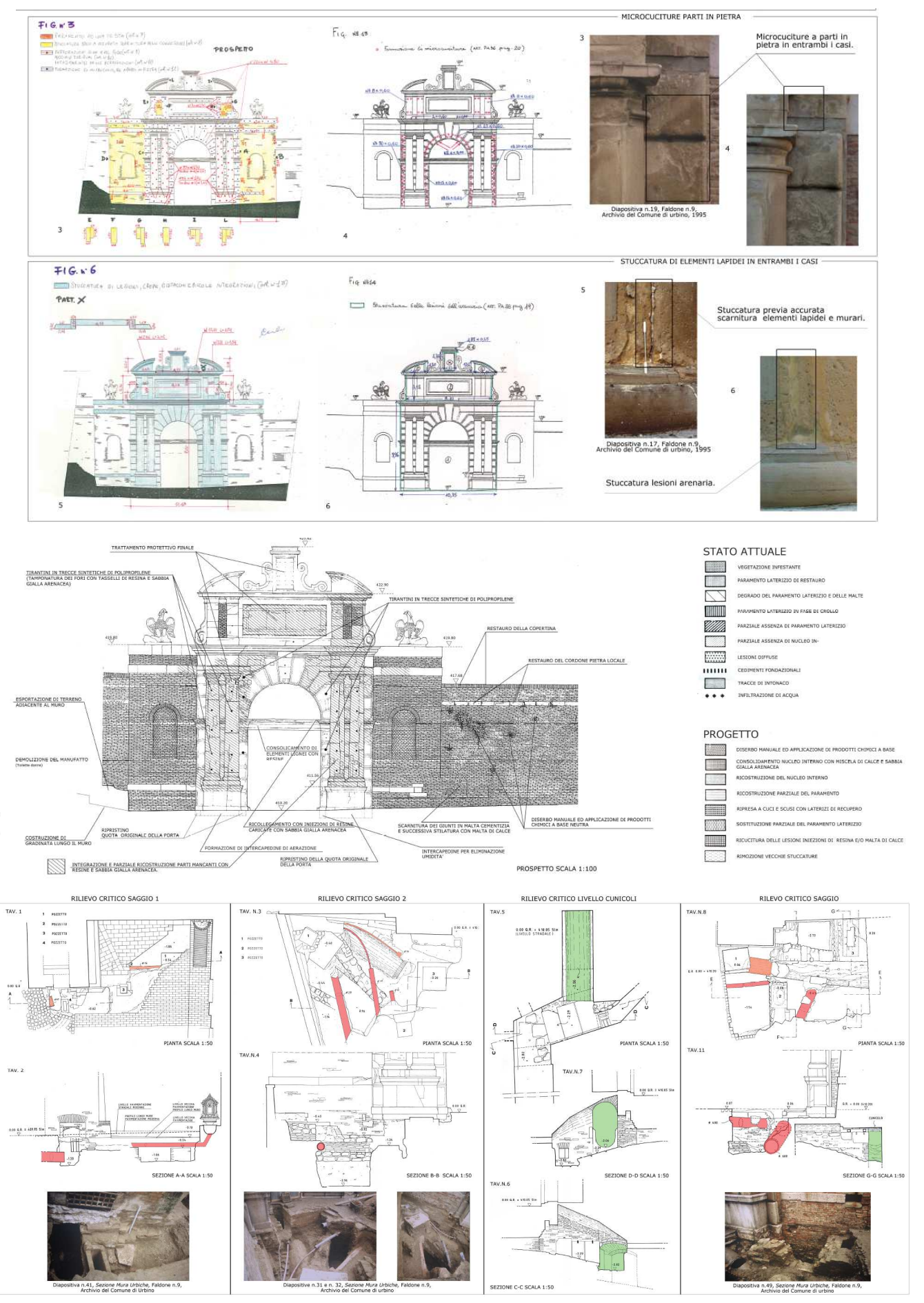

Fig. 8. Analisi dello stato attuale, indagini scientifiche e interventi di restauro della seconda metà del '900 (Archivio del Comune di Urbino). 


\section{Porta Valbona e la gestione dei dati}

Le restituzioni grafiche, tutta la documentazione iconografica e informativa sulle caratteristiche materiche e sui vari rilievi eseguiti nel tempo sulla porta e sull'evoluzione stessa della sua conformazione, sono andati a integrarsi in un sistema GIS che consente l'analisi e la gestione in ambiente bidimensionale e tridimensionale. Il progetto porta a una accurata definizione di Porta Valbona, attraverso un processo ben definito che consente di effettuare uno studio conoscitivo dettagliato sulle caratteristiche storiche, artistiche e architettoniche del manufatto e della sua evoluzione nei secoli, arrivando a un completo percorso di rilievo e gestione delle caratteristiche odierne. Grazie alla capacità di organizzare tutti i dati in un unico sistema Geodatabase le informazioni relative allo stato di conservazione e degrado, restauri effettuati, i confronti con altre tipologie simili di porte, l'evoluzione costruttiva, la mappatura delle patologie, interventi prece- denti e previsti, diventano tutti layer interrogabili e sovrapponibili che possono venire sottoposti a interrogazioni e analisi statistiche, elemento fondamentale per un'accurata manutenzione programmata. L'immagine sottostante mostra l'approccio metodologico utilizzato con il GIS per gestire le varie componenti di Porta Valbona: in alto le mura georeferenziate sono suddivise in segmenti che identificano gli elementi di interesse, elencati in tabella degli attributi con il campo NomeID, che funge da campo chiave per il database relazionale con cui spostarsi direttamente al prospetto di interesse. Ogni elemento del prospetto è associato a una serie di informazioni in tabella che possono fornire una serie di visualizzazioni differenti, con la creazione di una cartografia dinamica, che consente anche l'elaborazione di grafici e analisi statistiche. La gestione e l'interrogazione finale di tutto il progetto avviene, dopo opportuna sistemazione e georeferenziazione, in ambiente 3D, dove piante, modelli e prospetti coesistono. (Figg. 9 e 10).
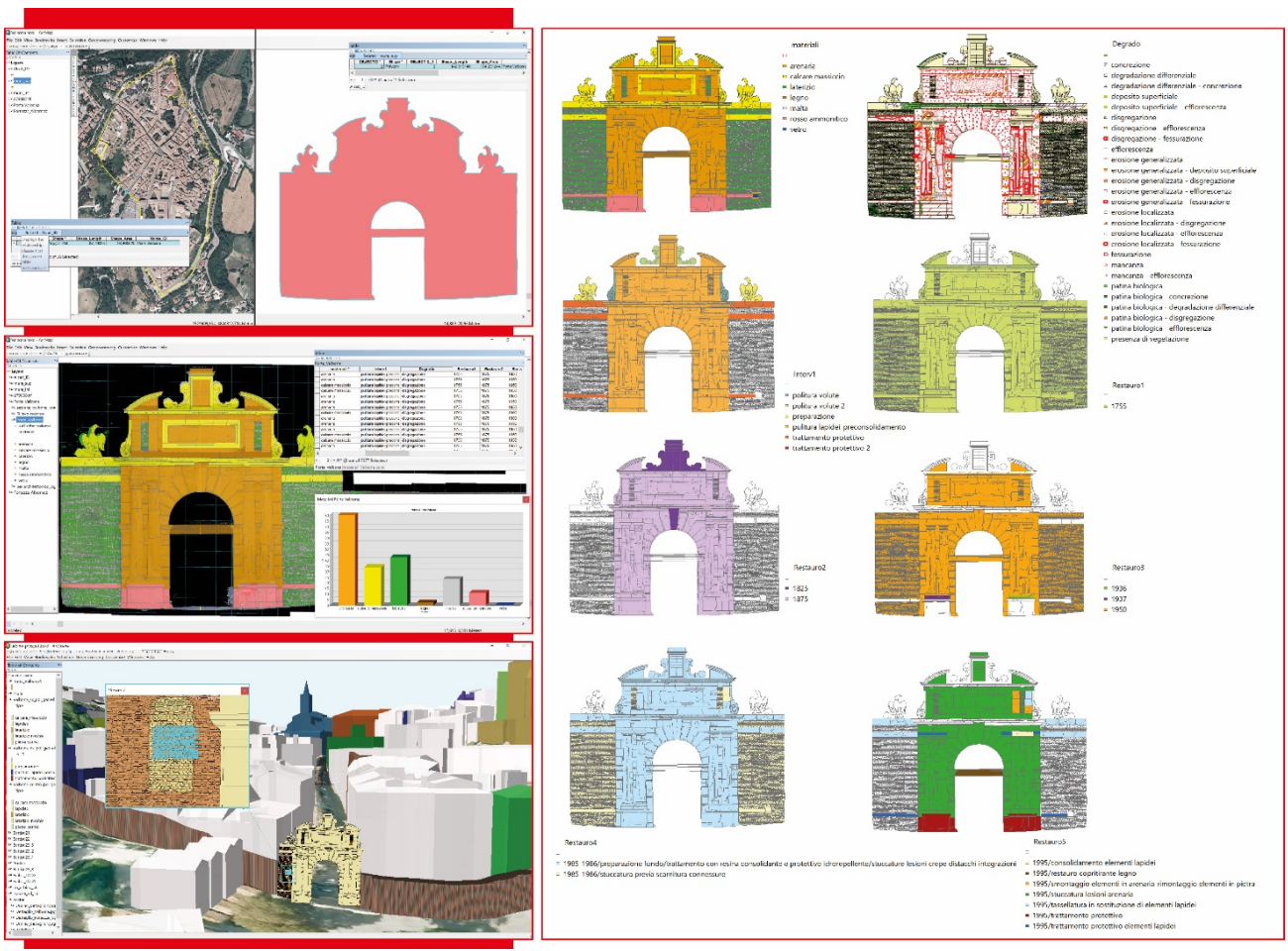

Fig. 9. Geodatabese su Porta Valbona. 

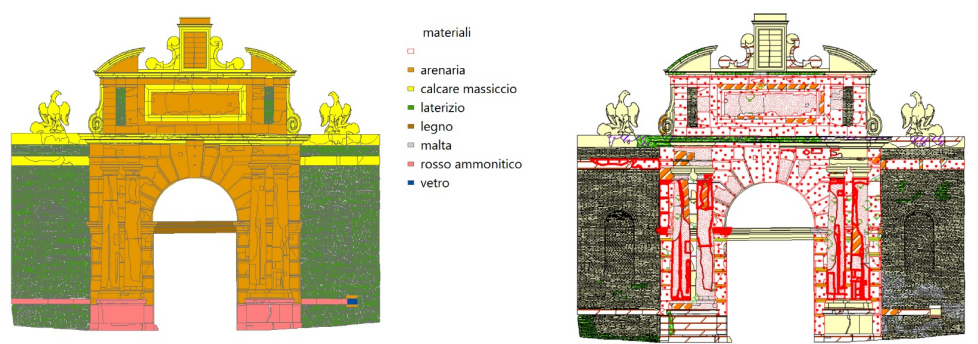

Degrado

$\varpi$ concrezione

$\square$ degradazione differenziale

n degradazione differenziale - concrezione

- deposito superficiale

- deposito superficiale - efflorescenza

a disgregazione

a disgregazione - efflorescenza

a disgregazione - fessurazione

$\square$ efflorescenza

$\square$ erosione generalizzata

a erosione generalizzata - deposito superficiale

* erosione generalizzata - disgregazione

$\checkmark$ erosione generalizzata - efflorescenza

a erosione generalizzata - fessurazione

a erosione localizzata

at erosione localizzata - disgregazione

- e rosione localizzata - efflorescenza

$\square$ erosione localizzata - fessurazione
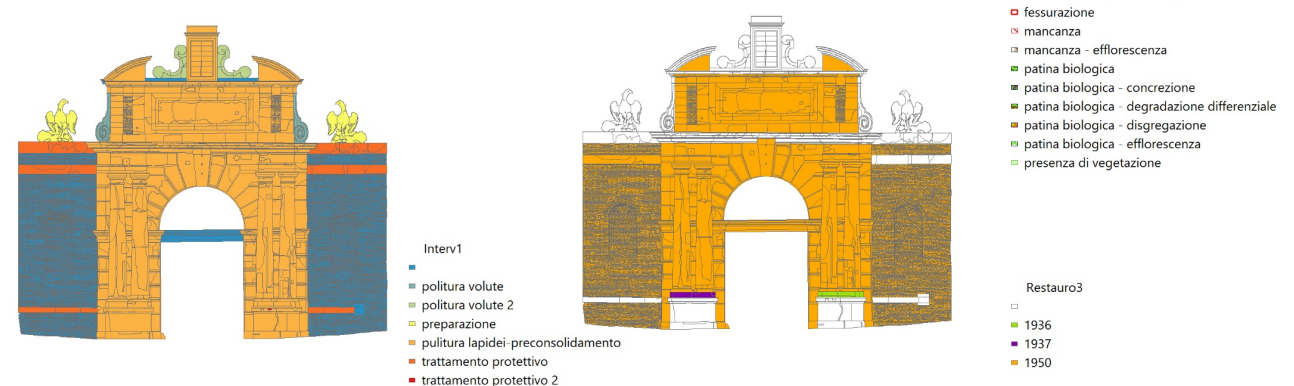

Fig. 10. Geodatabese su Porta Valbona alcuni dettagli.

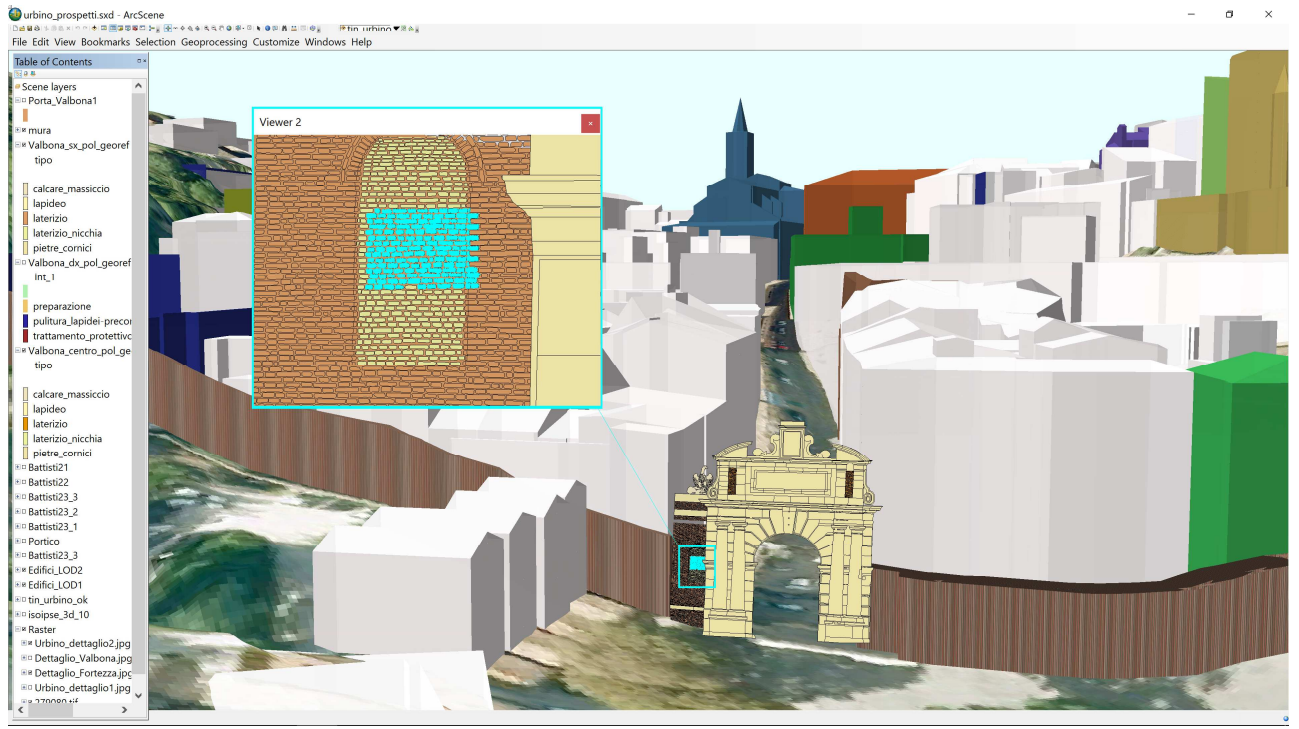

Fig. 11. Geodatabese su Porta Valbona elaborazioni 3D attraverso Arc-Scene.

\section{Conclusioni}

A partire da questo esempio sono in via di definizione una serie di linee guida su come affrontare e gestire le trasformazioni dell'immagine urbana e ambientale in analisi diacroniche, associate ad un'analisi e gestione di dettaglio dell'organizzazione urbana odierna, sfruttando le

caratteristiche 3D in maniera oculata e mirata ad un determinato risultato, che portino a una visione d'insieme della città nelle sue connotazioni più profonde caratterizzate dall'appartenenza alla lista dei siti patrimonio dell'umanità dell'UNESCO. 


\section{Bibliography}

Baratin, L.; Bertozzi, S.; Moretti, E. (2014). "Cultural Heritage: restituzione reale di prospetti e piante in ArcScene", in Atti della 15 Conferenza Italiana Utenti ESRI, Supplemento a GEOMEDIA, vol. 2.

Baratin, L.; Bertozzi, S.; Moretti, E. (2015a). "GIS intelligence for a cutting-edge management of 3D Cities", in Proceedings of Digital Heritage - International Congress, Granada.

Baratin, L.; Bertozzi, S.; Moretti, E. (2015b). "The Geomorphological transformations of the City of Urbino: the design of the city analysed with GIS tools", SCIRES it, SCIentific RESearch and In-formation Technology, 1, 5, pp. 41-60.

Baratin, L.; Bertozzi, S.; Moretti, E. (2016a). "The Fortification System of the City of Urbino: The Case Study of Valbona Gate from 3D Surveys to GIS Applications for Dynamic Maps", in Digital Heritage. Progress in Cultural Heritage:Documentation, Preservation and Protection, pp. 645-656.

Baratin, L.; Bertozzi, S.; Moretti, E. (2016b). "L'evoluzione delle trasformazioni di un centro storico tramite GIS: la città di Urbino", in Atti della Conferenza ESRI Italia, Roma.

Baratin, L.; Bertozzi, S.; Moretti, E. (2016c). "Le trasformazioni della città di Urbino durante il periodo dei Montefeltro: tecniche innovative per lo studio delle trasformazioni urbane", in Cennamo, G.M. ed., Processi di analisi per strategie di valorizzazione dei paesaggi urbani. I luoghi storici tra conservazione e innovazione. Atti del convegno Roma, 29 Gennaio 2016, Ermes, Servizi editoriali integrati S.r.l., Ariccia, pp. 105-115.

Baratin, L.; Cattaneo, A. (2018). "The walls of Urbino: a project of restoration, conservation and enhancement integrated in the historical city", in BHM\&P'18 Built Heritage Management and Presentation.

Baratin, L.; Cattaneo, A.; Gasparetto, F.; Moretti, E.; Lonati, S. (2019). "Documenting the conservative evolution of the city walls thanks to the integration of digital systems of various typologies. The case study of Valbona gate", The international archives of the photogrammetry, remote sensing and spatial information sciences, XLII2/W11, pp. 167-172.

Benevolo, L.; Boninsegna, P. (2000). Urbino, Laterza, Roma-Bari.

Bertozzi, S.; Moretti, E. (2015). "Gestione integrata dei rilievi del Portico di San Francesco ad Urbino", in Baratin, L.; Acierno, M.; Muratore, O., eds., Strumenti e Metodi per la Conservazione e la Valorizzazione dei Beni Culturali, Il Gabbiano, Ancona, pp. 114-121.

Mazzini, F. (2000). Urbino: i mattoni e le pietre, Argalìa, Urbino. 